\title{
Interacting electrons in a semiconducting carbon nanotube dot: a 2-band approach
}

\author{
Mervyn Roy ${ }^{\mathrm{a}, 1}$, and P. A. Maksym ${ }^{\mathrm{a}}$ \\ ${ }^{a}$ Dept. Physics and Astronomy, University of Leicester, Leicester, LE1 7RH, UK.
}

\begin{abstract}
A new 2-band effective mass theory for the interacting electron states in a carbon nanotube quantum dot is outlined. The states of 2 interacting electrons are calculated by exact diagonalisation of the 2-band Hamiltonian. A range of different nanotube and dot parameters are investigated and, interestingly, the 2-electron ground states are found to be ferromagnetic.
\end{abstract}

Key words: Nanotubes, quantum dots, electron correlations

PACS: 73.21.La, 73.23.Hk, 73.43.Cd, 73.63.Fg

\section{Introduction}

Semiconductor nanotube (NT) quantum dots are 1D artificial atoms. Recently, single electron transistors have been built from these novel quantum dots [1], and detailed experimental measurements of the dot addition energy have been made [1]. However, despite these advances, the physics of semiconductor NT dots is not well understood. We have developed a new 2-band effective mass approach to describe the states of a few interacting electrons in these dots. Our motivation is to understand the interacting states for a wide range of both NT and dot parameters. In this paper we focus on the effect of the band coupling on the 2-electron states and, interestingly, we find that when the band coupling is included, the 2-electron ground state can be ferromagnetic.

We consider gated NT dots similar to those studied by Jarillo-Herrero et al [1]. In our dot model electrons are confined electrostatically near the tube centre and the $1 \mathrm{D}$ dot confinement potential is harmonic $[1,2]$. We can write down an effective mass theory to describe the states of such a dot and there are some

$\overline{1}$ Corresponding author. E-mail: mr6@le.ac.uk 
new features that arise from the NT band structure. In all semiconducting NTs there are 2 equivalent conduction bands with minima at $\pm \mathbf{k}_{\mathbf{o}}$. Within the zone folding approximation these arise from the equivalence of the $K$ and $K^{\prime}$ points in the graphene zone. The Coulomb interaction couples these bands and, to investigate the effect of this coupling, we derive a new effective mass theory from the Hamiltonian of a graphene sheet.

The graphene Bloch states can be written in 2 component form in terms of the Bloch functions $\phi_{A}$ and $\phi_{B}$ of the A,B sub-lattices of graphene, $\psi_{\mathbf{k}}=$ $(1 / \sqrt{2}) e^{\imath \mathbf{k} \cdot \mathbf{r}}\left(\phi_{A}+(-1)^{n} e^{\imath \theta_{\mathbf{k}}} \phi_{B}\right)$ where $n=1,0$ for the valence or conduction band, and $\theta_{\mathbf{k}}$ is a phase factor. We construct our NT states from these two component Bloch functions. The key components of our theory are as follows. First, we include the Coulomb coupling between the NT states near $K$ and $K^{\prime}$. Secondly, we ignore the coupling with the valence band, and this is valid because the dot energy scales $(\hbar \omega \sim$ meV) are much smaller than the band gap in a typical semiconductor $\mathrm{NT}(\sim \mathrm{eV})$. The net result is a 1 dimensional, 2-band effective mass theory with a modified Coulomb interaction. We obtain additional terms in the interaction depending on the separation, $\delta_{k}=1 /(3 R)$, in $k$-space between the NT conduction band minima and the graphene $K$ or $K^{\prime}$ points ( $R$ is the tube radius). The total interaction is $u=\left(2 v_{0}(z, R)+v_{0}(z-\right.$ $\left.3 R, R)+v_{0}(z+3 R, R)\right) / 4$ for intraband scattering and $u=S_{\mathbf{K}-\mathbf{K}^{\prime}}^{2}\left(2 v_{p}(z, R)-\right.$ $\left.v_{p}(z-3 R, R)-v_{p}(z+3 R, R)\right) / 4$ for interband processes, where $z$ is the electron separation, $S_{\mathbf{K}-\mathbf{K}^{\prime}}^{2}$ is an atomic form factor $\left(0 \leq S^{2} \leq 1\right)$ and the Coulomb term, $v_{q}$, is defined in section 2 . The integer, $p$, measures the separation in $\mathbf{k}$ between the two NT conduction bands in the direction parallel to the chiral vector of the tube. In a semiconducting $(n, 0)$ zigzag tube, $p=2(n \pm 1) / 3$.

\section{Results}

We diagonalise the NT quantum dot Hamiltonian, $H=\sum_{i j}\left\langle i\left|h_{o}\right| j\right\rangle c_{i}^{\dagger} c_{j}+$ $(1 / 2) \sum_{i j k l}\langle i j|u| k l\rangle c_{i}^{\dagger} c_{j}^{\dagger} c_{k} c_{l}$, to an accuracy of better than $0.1 \%$. We are interested in a wide range of $\mathrm{NT}$ dots with many different parameters: for example, different $\hbar \omega$, dielectric constant $\epsilon_{r}$, effective mass $m^{*}$ or tube radius $R$. By writing the dot Hamiltonian in dimensionless form we can describe this parameter space in terms of just 2 dimensionless parameters $(\alpha, \beta)$ when we neglect the interband coupling and 3 parameters $(\alpha, \beta, p)$ when we include it. We choose the energy unit to be $E_{u}=\sqrt[3]{\rho^{2} m^{*} \omega^{2} / \epsilon_{r}^{2}}$ and the length unit to be $\lambda=\sqrt[3]{\rho /\left(\epsilon_{r} m^{*} \omega^{2}\right)}$ where $\rho=e^{2} /\left(4 \pi \epsilon_{o}\right)$. Then, with $x=z / \lambda$, $\alpha^{3}=\left[\rho^{2} m^{*} /\left(\hbar^{3} \epsilon_{r}^{2} \omega\right)\right]^{2}$ and $\beta=R / \lambda$, we have $h_{o}=(-1 / 2 \alpha) \nabla_{x}^{2}+x^{2} / 2$ and $v_{q}(x, \beta)=(1 / 2 \pi) \int\left(\cos (2 q \phi) / \sqrt{x^{2}+4 \beta^{2} \cos ^{2}(\phi / 2)}\right) d \phi$. As we constrain the parameter space by $1 \leq \epsilon_{r} \leq 7$ and $1 \leq \hbar \omega \leq 50 \mathrm{meV}$ the range of allowed $\alpha$ depends on $\beta$. 

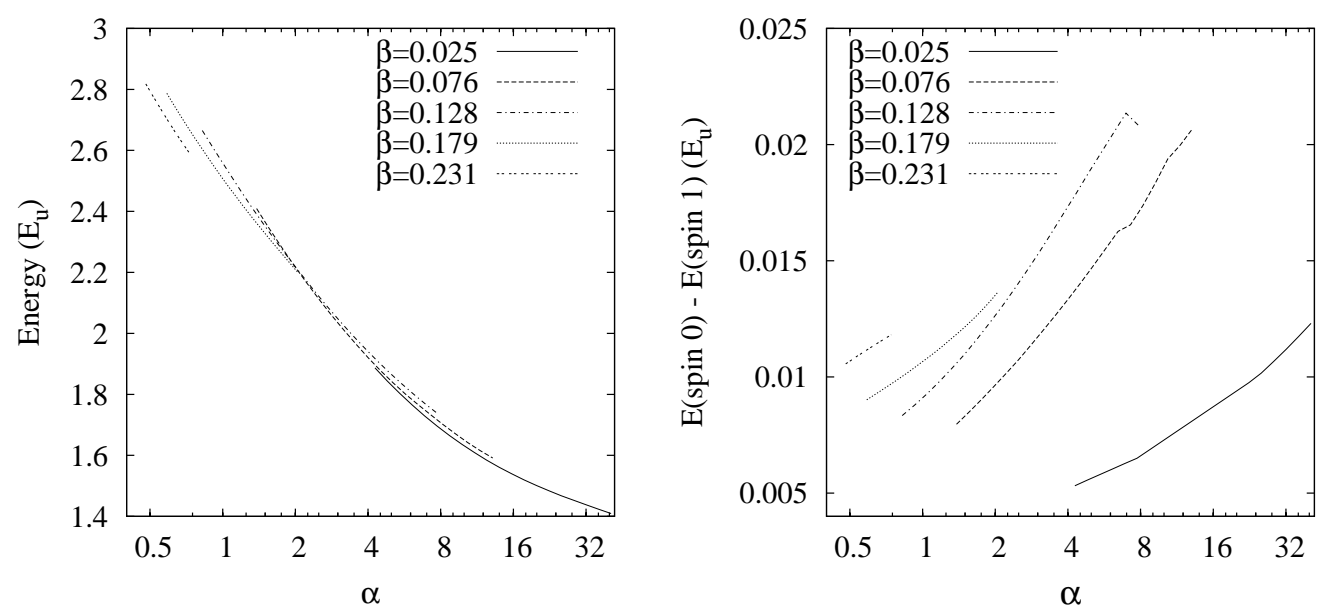

Fig. 1. Left: 4-fold degenerate, 2 electron ground state energy $\left(S^{2}=0\right)$. Right: Splitting of spin 1 and spin 0 states caused by the interband coupling $\left(p=3, S^{2}=1\right)$.

The ground state energy (Fig 1 (left)) decreases with increasing $\alpha$ as we would expect: in the dimensionless Hamiltonian $\alpha$ is analogous to a mass. When we neglect the coupling between the two bands, the band index acts like a pseudospin and the ground state is 4-fold degenerate. When we include the coupling term the spin 0 and spin 1 ground states are split. Fig. 1 (right) shows the splitting for a $(10,0)$ NT $(p=3)$ and, by assuming $S^{2}=1$, we have calculated the maximum possible values for this splitting. The spin 1 state has the lowest energy because only the spin 1 state gets any benefit from the electron exchange. This does not contradict the Lieb-Mattis [3] theorem because our Hamiltonian includes 2-bands. We have also calculated the splitting across the parameter space for $p=12$, a $(35,0) \mathrm{NT}$. In this case, the splitting is of the order of the numerical error in the energy $(0.1 \%)$.

Over the parameter space that we have investigated, the 2 electron ground state is ferromagnetic. The magnitude of the spin 1 and spin 0 splitting is small ( $\sim 1 \%$ of the ground state energy) but it is largest when $p$ is small (small NTs) and smallest when the electrons are either very strongly (large $\alpha$ ) or very weakly ( small $\alpha$ ) correlated. This work was supported by the UK EPSRC and was performed using the University of Leicester Mathematical Modelling Centre's Supercomputer.

\section{References}

[1] P. Jarillo-Herrero, S. Sapmaz, C. Dekker, L. P. Kouwenhoven and H. S. J. van der Zant, Nature 429, 389 (2004).

[2] M. Roy and P. A. Maksym, Phys. Stat. Sol. (c) 3, 3959 (2006).

[3] E. Lieb and D. Mattis, Phys. Rev. 125, 164 (1962). 